\title{
855 DEVELOPMENT OF A NOVEL ANTI-MULTIPLE MYELOMA CHIMERIC ANTIGEN RECEPTOR T CELL THERAPY
}

Chuck Hay*, Mary Faber, Kemi Adeyanju, Jeffrey Medin. Medical College of Wisconsin, Wauwatosa, WI, USA

Background Multiple myeloma is a cancer of plasma cells, wherein the plasma cells begin outgrowing and even suppressing the growth of normal hematopoietic-lineage cells in the blood marrow. It is estimated that nearly 35,000 new cases of multiple myeloma will be diagnosed each year, and over 12,000 individuals will die from multiple myeloma in 2021. The current overall 5-year survival rate of 54\% stresses the need for alternative treatment strategies. A chimeric antigen receptor $\mathrm{T}$ cell (CAR-T) therapy that targets multiple myeloma surface proteins may have the potential to improve this survival rate.

Methods A commonly associated multiple myeloma antigen was selected, and the extracellular domain of the protein was expressed in an Expi293 cell culture system. The multiple myeloma protein was expressed with and purified by a Hisx6 tag. The purified protein was used to pan a specific $\mathrm{scFv}$ phage display library that was generated uniquely for our lab from multiple blood donors. Several clones were identified and sequenced. One clone was selected and verified to be capable of binding the multiple myeloma antigen via ELISA. This anti-multiple myeloma $\mathrm{scFv}$ was then subcloned into CAR-T plasmids. This is a second-generation CAR-T using a 4$1 \mathrm{BB}$ co-stimulator domain and a $\mathrm{CD} 3 \zeta$ intracellular signaling domain was used for this therapy. Further, a cell-fate control gene, LNGFR $\Delta$ Tmpk, was added to the plasmid to combat potential graft versus host disease. CAR-T and lentivirus (LV) plasmids were transfected into HEK cells to produce anti-multiple myeloma CAR-T LV.

Results The anti-multiple myeloma CAR LV was transduced into HEK cells and titered via qPCR, which showed the LV prep produced $3.1 \mathrm{E} 8 \mathrm{IU} / \mathrm{ml}$. qPCR also showed there to be 0.407 vector copies per cell on average when using an MOI of 3. Our CAR-T LV was then used to transduce Jurkat cells at an MOI of 3. Using anti-Fab and anti-protein $\mathrm{L}$ antibodies, the transduced cells were shown to be expressing the antimultiple myeloma CAR-T by flow cytometry. After confirming successful transduction and expression, transduced Jurkats (and controls) were co-cultured with multiple myeloma cell lines and incubated for 24 hours. ELISA testing for IFN- $\gamma$ was performed on the resulting supernatants; specific engagement was demonstrated.

Conclusions These results show that our anti-multiple myeloma CAR is successfully expressed and is functional. Our future goal is to successfully transduce our anti-multiple myeloma CAR LV into primary $\mathrm{T}$ cells and test for functionality in these cells before transitioning into in vivo models.

http://dx.doi.org/10.1136/jitc-2021-SITC2021.855 